\title{
Relación de autores que se mencionan
}

Dr. H. Baas

Klinikum d. Univ. Frankfurt/M.

Abt. f. Neurologie

Zentrum d. Neurologie

u. Neurochirurgie

Schleusenweg 2-16

D-6000 Frankfurt/Main 71

Prof. Dr. P.-A. Fischer

Klinikum d. Univ. Frankfurt/M.

Abt. f. Neurologie

Zentrum d. Neurologie

u. Neurochirurgie

Schleusenweg 2-16

D-6000 Frankfurt/Main 71

Dr. B. Frieling

Schering Aktiengesellschaft

Postfach 650311

D-1000 Berlin 65

Dr. R. Horowski

Schering Aktiengesellschaft

Postfach 650311

D-1000 Berlin 65
Prof. Dr. P. Riederer

Psychiatr. Univ.- und Poliklinik

Füchsleinstraße 15

D-8700 Würzburg

Prof: Dr. U. K. Rinne

University of Turku

Dept. of Neurology

SF-20 520 Turku 52

Dr. I. Suchy

Schering Aktiengesellschaft

Postfach 650311

D-1000 Berlin 65

Dr. G. Ulm

Paracelsus Elena-Klinik

Klinikstraße 16

D-3500 Kassel

Dr. H. Wachtel

Schering Aktiengesellschaft

Postfach 650311

D-1000 Berlin 65

Prof. Dr. H. Przuntek

Neurolog. Univ.-Klinik

im St. Josef-Hospital

Gudrunstraße 56

D-4630 Bochum 
